IRA-International Journal of Technology \& Engineering

ISSN 2455-4480; Vol.10, Issue 03 (March, 2018)

Pg. no. 41-54.

Institute of Research Advances

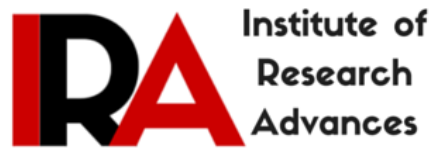

https://research-advances.org/index.php/IRAJTE

\title{
Air Permeability of Thick Foams: Flow Numerical Simulations
}

Karel Adámek

VÚTS Liberec, Svárovská 619, CZ-46119 Liberec, Czech Republic.

Type of Review: Peer Reviewed.

DOI: http://dx.doi.org/10.21013/jte.v10.n3.p2

\section{How to cite this paper:}

Adámek, K. (2018). Air Permeability of Thick Foams: Flow Numerical Simulations. IRA International Journal of Technology \& Engineering (ISSN 2455-4480), 10(3), 41-54. doi:http://dx.doi.org/10.21013/jte.v10.n3.p2

(c) Institute of Research Advances.

\section{(cc) EY-NC}

This work is licensed under a Creative Commons Attribution-Non Commercial 4.0 International License subject to proper citation to the publication source of the work.

Disclaimer: The scholarly papers as reviewed and published by the Institute of Research Advances (IRA) are the views and opinions of their respective authors and are not the views or opinions of the IRA. The IRA disclaims of any harm or loss caused due to the published content to any party.

Institute of Research Advances is an institutional publisher member of Publishers Inter Linking Association Inc. (PILA-CrossRef), USA. The institute is an institutional signatory to the Budapest Open Access Initiative, Hungary advocating the open access of scientific and scholarly knowledge. The Institute is a registered content provider under Open Access Initiative Protocol for Metadata Harvesting (OAI-PMH).

The journal is indexed $E^{\circ}$ included in WorldCat Discovery Service (USA), CrossRef Metadata Search (USA), WorldCat (USA), OCLC (USA), Open J-Gate (India), EZB (Germany) Scilit (Switzerland), Airiti (China), Bielefeld Academic Search Engine (BASE) of Bielefeld University, Germany, PKP Index of Simon Fraser University, Canada. 


\begin{abstract}
From measured data are determined permeability parameters of thick perforated foam samples, used as car seats cushions. Parameters are used for numerical flow simulations in foam samples. Model of detailed geometry gives good view about detailed flow field (pressure and velocity) in foam volume, influenced by perforations and grooves. However, simulated flow is several times different from measured one. The main flow is through perforations (99\%) and flow through foam is of two orders lower. Using homogenous geometry with "averaged" permeability parameters, evaluated from measured values, the coincidence of measured and simulated flow is very good, difference of 1-5\%. However, it is not possible to get any details of flow in foam volume. Using inlet layer, the flow is decreasing, first in perforations and the ratio between perforation and foam flows is more balanced.
\end{abstract}

Keywords: Air permeability, flow numerical simulation, thick foams

\title{
1. APPLICATION OF MEASURED DATA
}

The method used for former numerical simulation of air permeability in thin perforated foams of $5 \mathrm{~mm}$ [1] is used here for thick perforated and grooved foams of $30 \mathrm{~mm}$, too, to get an opinion about air flow through such complicated thick layer.

\subsection{Measured samples}

The Fig. 1 presents the appearance of foam samples, used for measuring [2]. Main dimensions: sample area 165 $\mathrm{cm}^{2}$ (diameter of $145 \mathrm{~mm}$, thickness of $30 \mathrm{~mm}$ ), diameter of perforations 6-12-18 mm, grooves $5 \mathrm{~mm}$ width, 15 $\mathrm{mm}$ depth, pattern of $38 \times 44 \mathrm{~mm}$.

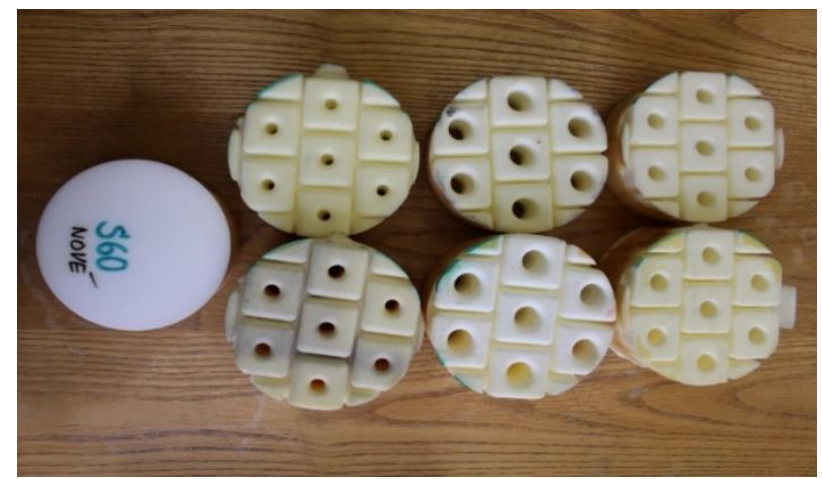

Fig. 1:Real samples of foams

Note 1: Concerning the terminology, the term ,permeability“, used in textile technologies, is defined as volume flow $\left(\mathrm{m}^{3} / \mathrm{s}\right)$ through the sample area $\left(\mathrm{m}^{2}\right)$,i.e. $\mathrm{m} / \mathrm{s}=\mathrm{m}^{3} / \mathrm{s} \cdot \mathrm{m}^{2}$. In the fluid mechanics the same parameter is known as ,,volume flow density“،

It should be better to use the „mass flow density” $\left(\mathrm{kg} / \mathrm{s} . \mathrm{m}^{2}\right)$, which is independent on state quantities (pressure, temperature). This term is used in numerical flow simulations below and in the fluid mechanics, too. Therefore, for later comparison of measured and simulated flows the measured volume flows $(\mathrm{ml} / \mathrm{s})$ are recalculated into mass flows $(\mathrm{kg} / \mathrm{s})$.

\subsection{Discussion of measured data}

All solved samples - perforated and grooved - can be regarded as the combination of three kinds of many elementary flow resistances in parallel connection: empty holes, full foams and combined serial connections of empty groove with foam (both as half thicknesses).

The pressure gradients, used for measuring of foams, are used for numerical flow simulations, too.

So-called flow characteristics

$$
\mathrm{V}(\mathrm{ml} / \mathrm{s})=\mathrm{f}(\Delta \mathrm{p}(\mathrm{Pa}))
$$


are given by measuring [2], where F0 is the full foam and F1-F2-F3 are perforated foams of different holes diameter. For next determination of permeability parameters (see the section 1.2) the characteristics are recalculated and inverted in so-called resistance characteristics

$$
\Delta \mathrm{p}(\mathrm{Pa})=\mathrm{f}(\mathrm{w}(\mathrm{m} / \mathrm{s}))
$$

Note 2: As an information, only, the Fig. 2 shows the typical velocity field in three used hole diameters of 6, 12 and $18 \mathrm{~mm}$ in the foam thickness of $30 \mathrm{~mm}$, for pressure difference of 1,1 Pa. It is known, that

- the inlet resistance and friction resistance along the channel is relatively more significant for smaller diameters, because the boundary layer thickness is relatively thick in small diameters (it depends on medium viscosity, wall roughness and not on the diameter,

- the creation of fully developed velocity profile in the tube needs the length of the tube of 10 diameters as minimum, in solved cases there is $\mathrm{L} / \mathrm{d}=0.6 \ldots 5.0$, only.
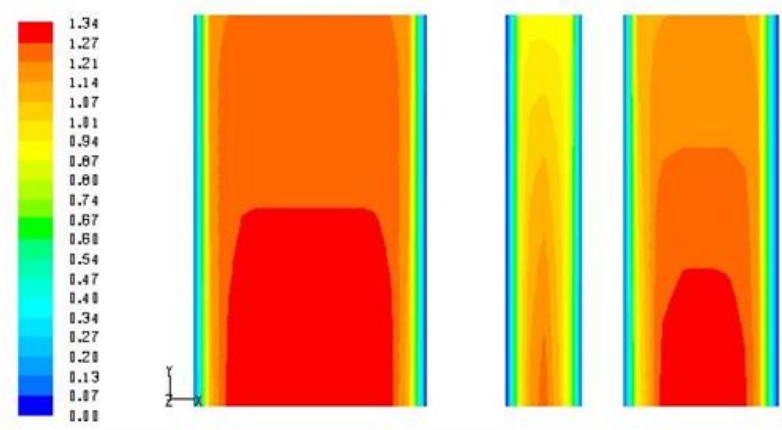

Fig. 2: Typical velocity fields $(\mathrm{m} / \mathrm{s})$ in empty holes of various diameters

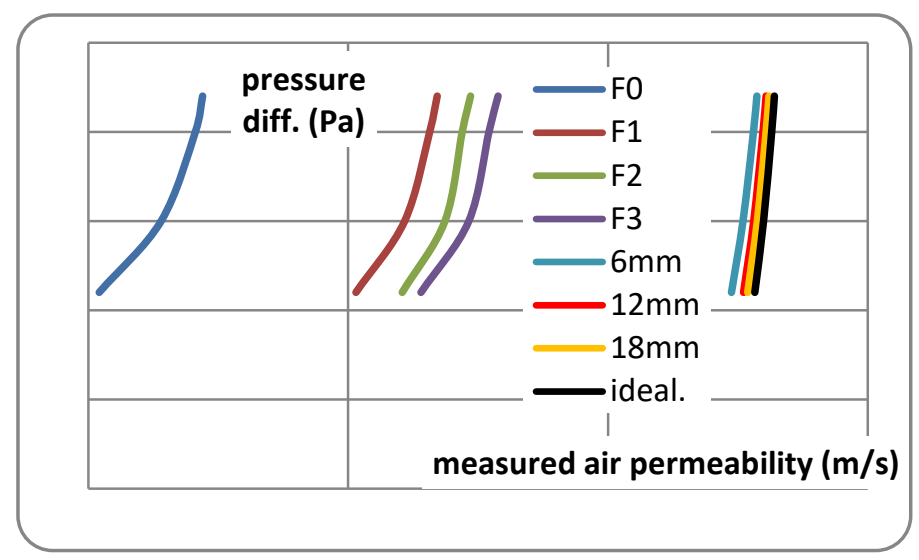

Fig. 3: Inverted and recalculated characteristics in semi-logarithmic scale

Together with the characteristic of ideal flow

$$
\Delta p=\rho / 2 \cdot w^{2}
$$

all measured and simulated results presents the common graph Fig. 3.

Using the semi-logarithmic scale it is visible, that the measured permeability of the full foam F0 is of two orders lower than of perforated foams F1, F2, F3 and simulated "permeability" of empty holes after the Fig. 2 is of 3 next orders higher. All resulting data see the Tab. 1. 
Table 1: Data comparison - given, measured, simulated

\begin{tabular}{|c|c|c|c|c|c|}
\hline & & \multicolumn{3}{|c|}{ abs. } & remark \\
\hline & foam: & $\mathrm{F} 1$ & $\mathrm{~F} 2$ & F3 & \\
\hline \multirow{2}{*}{$\begin{array}{l}\text { perforations } \\
\text { diameter }\end{array}$} & $\mathrm{mm}$ & 6 & 12 & 18 & \\
\hline & relat. & 1 & 2 & 3 & \\
\hline \multirow{2}{*}{$\begin{array}{l}\text { perforations } \\
\text { area }\end{array}$} & $\mathrm{mm}^{2}$ & 36 & 144 & 324 & \\
\hline & relat. & 1 & 4 & 9 & \\
\hline \multirow{2}{*}{$\begin{array}{l}\text { measured } \\
\text { air flow. }\end{array}$} & $\mathrm{ml} / \mathrm{s}$ & 80 & 145 & 235 & \\
\hline & relat. & 1.00 & 1.81 & 2.94 & $\sim \mathrm{d}^{1}$ \\
\hline \multirow{2}{*}{$\begin{array}{l}\text { simulated } \\
\text { volume } \\
\text { flow }\end{array}$} & $\mathrm{ml} / \mathrm{s}$ & 38 & 187 & 443 & \\
\hline & relat. & 1.00 & 4.89 & 11.80 & $>d^{2}$ \\
\hline \multirow{2}{*}{$\begin{array}{l}\text { simulated } \\
\text { velocity } \\
\text { avg }\end{array}$} & $\mathrm{m} / \mathrm{s}$ & 0.87 & 1.14 & 1.36 & \\
\hline & relat. & 1.00 & 1.31 & 1.68 & $\sim \sqrt{d}$ \\
\hline
\end{tabular}

Note 3: Air permeability $(\mathrm{m} / \mathrm{s})$ measured as volume flow $(\mathrm{ml} / \mathrm{s})$ through given sample area $\left(\mathrm{m}^{2}\right)$ is proportional to given diameters $(\mathrm{m})$, according to laminar flow.

Simulated volume flow $\left(\mathrm{m}^{3} / \mathrm{s}\right)$ is proportional to $(1.22 \ldots 1.29) \cdot \mathrm{d}^{2}$, it is more than the proportion to the given area $\left(d^{2}\right)$.

Simulated outlet velocity $(\mathrm{m} / \mathrm{s})$ evaluated as vertex averaged values is proportional to the root of given diameters, only (probably $\sim \sqrt{d}$ ).

Mass flow is not evaluated, for small pressures is proportional to the volume flow.

Differences of simulated values could be the consequences of uneven flow in individual elements of the outlet area (foam, perforation, groove) and of determined procedure of evaluation. It should be to use very fine mesh and to evaluate the flow velocity and volume in each such element of the outlet area.

\subsection{Determination of permeability parameters}

The used method is described in [1, 3 etc.] for so-called 'porous jump' in one-dimensional flow and thin porous layer. Here is the method used for so-called 'porous zone', too, generally as the flow in 3-dimensional permeable volume (thick porous layer). Theoretical formulas for simulations [4] are analogous, so it is supposed to use the same procedure in 3D for homogenous permeability without important problems [1].

For testing the hypothesis here are used two foams, only, F0 (full foam of high flow resistance) and F1 (one of similar perforated foams after the Fig. 1). It supposes that for similar foams F2, F3 the method can be used, too, without problems. The procedure is demonstrated on the next serial of the Fig. 4 to Fig. 6. 


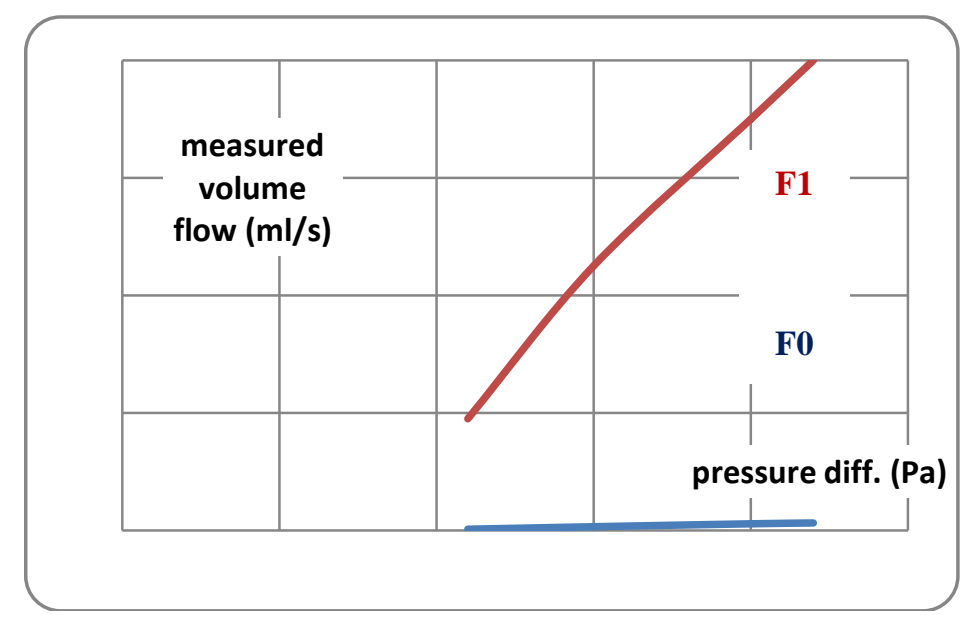

Fig. 4: Measured volume flows

Setting the pressure difference before and after the foam sample, the volume flow through foam sample is measured [2] and so-called flow characteristics (1) is created. Result must be converted into the resistance characteristic (2), see the Fig. 5. For it must be done the following:

- to convert the volume flow $(\mathrm{ml} / \mathrm{s})$ through the sample area $\left(\mathrm{m}^{2}\right)$ into flow velocity $(\mathrm{m} / \mathrm{s})$,

- to replace mutually axis $\mathrm{x}$ and $\mathrm{y}$ from previous graph and

- to create quadratic substitution, corresponding with (2), see the equations on the Fig. 5.

Note 4: The absolute term of the quadratic substitution should be $\mathrm{C}=0$ (for zero pressure difference the flow is zero, too). If $\mathrm{C} \neq 0$, it means that an error is occurred, here probably due to wrong zero setting or insensitivity of used measuring device, wrong reading, any leakage etc. It is the problem of very low used pressures - the error of 0.86 or $0.92 \mathrm{~Pa}$ approx. for used pressure differences from 1.1 to $2.2 \mathrm{~Pa}$ cannot be neglected.

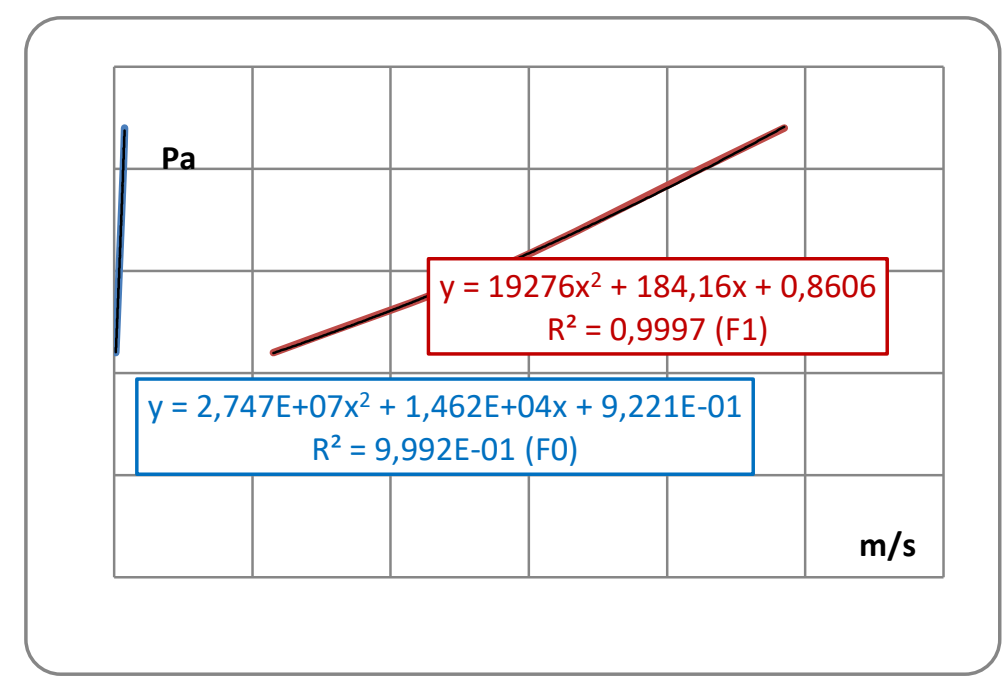

Fig. 5: Inverted and recalculated functions

Such error was not observable in similar solution [1]; the problem here must be solved in laboratory. For simulation purposes, only, here the measured values are simply shifted, to get $\mathrm{C} \approx 0$ in quadratic functions and to eliminate errors at very low pressures and flows, the result see the Fig. 6. 


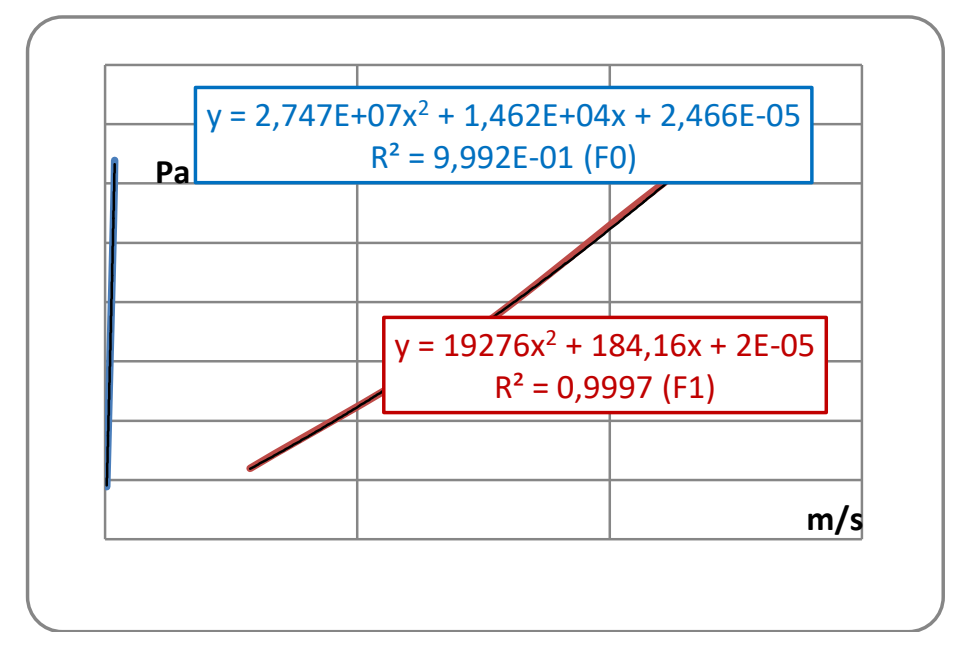

Fig. 6: Shifted graphs

After shifting, the measuring error (value of $\mathrm{C}$ in quadratic substitution) is going to zero and pressure values are shifting adequately down. Other parameters of quadratic substitutions A, B and $\mathrm{R}^{2}$ on graphs Fig. 5 and Fig. 6 remain the same.

Note5: The zero point could be added to measured data (not documented here). The results are very similar, the differences of parameters $\mathrm{A}, \mathrm{B}, \mathrm{C}$ and $\mathrm{R}^{2}$ can be neglected and their values can be used for definition of permeability parameters in numerical models.

Received parameters of quadratic substitutions from the Fig. 6 are in the Tab. 2 .

Table 2: Parameters of quadratic substitutions

$$
\begin{array}{ccccc}
\text { foam } & \text { A } & \text { B } & \text { C } & R^{2} \\
\text { F0 } & 2,747 \mathrm{e}+07 & 1,462 \mathrm{e}+4 & 2,466 \mathrm{e}-5 & 0,9992 \\
\text { F1 } & 19276 & 184,16 & 2,00-05 & 0,9997
\end{array}
$$

Comparing the formula for pressure resistance as function of velocity (parameters of quadratic substitutions A, B, C see the Tab. 2)

$$
\Delta \mathrm{p}=\mathrm{A} \cdot \mathrm{w}^{2}+\mathrm{B} \cdot \mathrm{w}+\mathrm{C}
$$

with formula for permeability [4]

$$
\Delta \mathrm{p}=\mathrm{C}_{2} \cdot \rho / 2 \cdot \mathrm{t} \cdot \mathrm{w}^{2}+\mu / \alpha \cdot \mathrm{t} \cdot \mathrm{w}
$$

we can determine two unknown permeability parameters $\mathrm{C}_{2}, \alpha$ from

$$
\begin{aligned}
& \mathrm{A}=\mathrm{C}_{2} \cdot \rho / 2 \cdot \mathrm{t} \\
& \mathrm{B}=\mu / \alpha \cdot \mathrm{t}
\end{aligned}
$$

as

$$
\begin{aligned}
& \mathrm{C}_{2}=2 \cdot \mathrm{A} /(\rho \cdot \mathrm{t}) \\
& \alpha=\mu /(\mathrm{B} \cdot \mathrm{t})
\end{aligned}
$$

where
$\mathrm{w}=\mathrm{V} / \mathrm{S}(\mathrm{m} / \mathrm{s})$
flow velocity
$\mathrm{V}\left(\mathrm{m}^{3} / \mathrm{s}\right)$
volume flow 


$\begin{array}{ll}\mathrm{S}\left(\mathrm{m}^{2}\right) & \text { flow cross-section } \\ \mathrm{t}(\mathrm{m}) & \text { layer thickness } \\ \rho\left(\mathrm{kg} / \mathrm{m}^{3}\right) & \text { medium density (for air } 1,2) \\ \mu(\text { Pa.s }) & \text { dynamic viscosity (for air } 1,806 \mathrm{e}-5) \\ \alpha\left(\mathrm{m}^{2}\right) & \text { unknown permeability parameter [4] } \\ \mathrm{C}_{2}(1 / \mathrm{m}) & \text { unknown permeability parameter [4] }\end{array}$

Resulting permeability parameters are in the Tab. 3.

Table 3: Evaluated permeability parameters

$\begin{array}{cccc}\text { foam } & \mathrm{C} 2(1 / \mathrm{m}) & \alpha\left(\mathrm{m}^{2}\right) & 1 / \alpha\left(1 / \mathrm{m}^{2}\right) \\ \text { F0 } & 1.526 \mathrm{e}+09 & 3.71 \mathrm{e}-11 & 2.698 \mathrm{e}+10 \\ \text { F1 } & 1.071 \mathrm{e}+06 & 2.94 \mathrm{e}-09 & 3.399 \mathrm{e}+08\end{array}$

Note 6: Another substitution $\Delta \mathrm{p}=\mathrm{f}\left(\mathrm{w}^{\mathrm{n}}\right)$ was used in [5], where $1<\mathrm{n}<2$. According to the above used formula it could be said that such function of pressure resistance contains any combination of the first and the second power of velocity.

\section{NUMERICAL SIMULATIONS}

Model for the first simulation (debugging, testing of developed procedure etc.) is created as F1, only, i.e. 'empty' volumes of grooves and holes are filled by air and 'full' volumes are filled by permeable basic foam of parameters F0. It supposes that the process for similar geometries of F2 and F3 is the same.

The values of all presented results are in the SI system (m/s for velocity, Pa for pressure, K for temperature etc.).

\subsection{Basic model}

Geometry on the Fig. 7 corresponds to the Fig. 1, sample diameter of $145 \mathrm{~mm}$ (sample area of $165 \mathrm{~cm}^{2}$ ). Mesh is created relative coarse, to shorten the solution time of many tested cases.

Laminar model is used due to low Reynolds number $(\operatorname{Re} \approx 100)$.

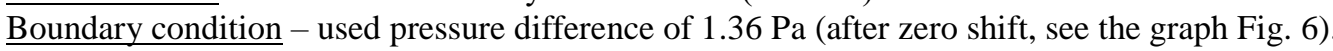

Next inlet and outlet volumes after and before the foam volume are not added here, so that the inlet and outlet boundary conditions are defined just at upper and lower surfaces of tested sample. Former models used in [1] contain such large inlet and outlet volumes, so there arise backflows and time of solution is prolonged, due to very low permeability of foam volume.

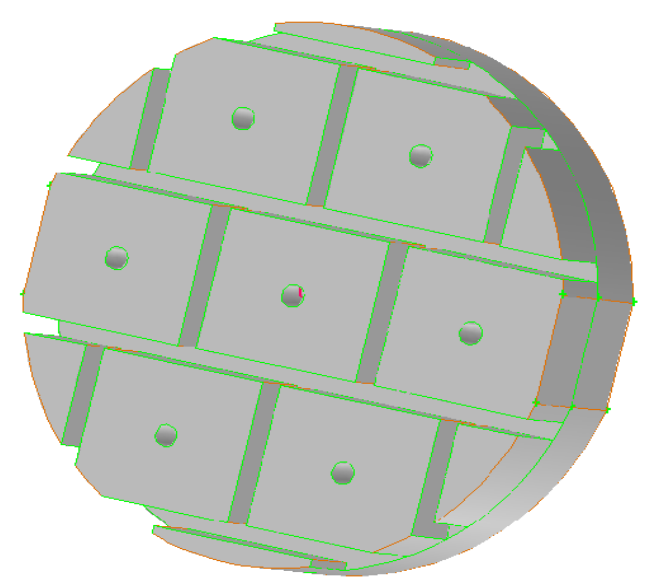

Fig. 7: Geometry of the basic model 
$\underline{\text { Results }}$

Next serial of flow fields presents main qualitative results. To get full details of individual flow fields, suitably different scales are used. In detailed geometry are well visible details of velocity and pressure fields, as for instance the induced flows in foam around holes and grooves and similarly. Our original results were presented in [6].
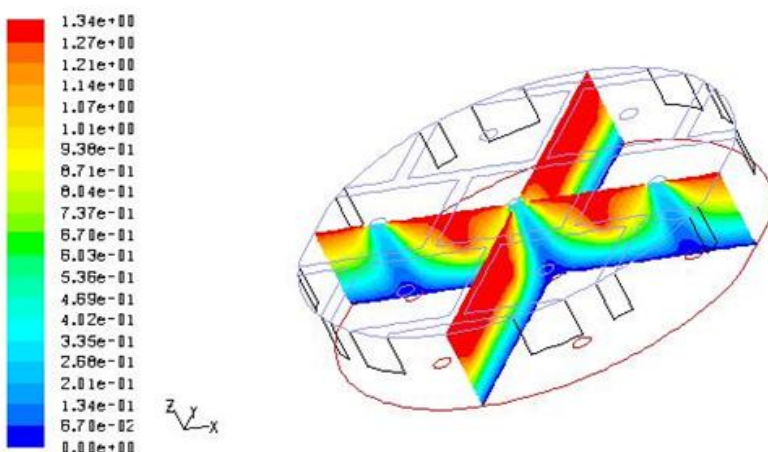

Fig. 8: Pressure field $(\mathrm{Pa})$ in axial cross sections
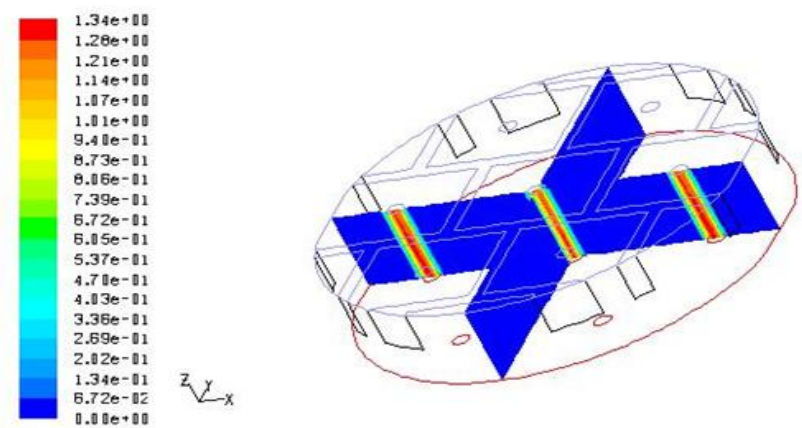

Fig. 9: Velocity field $(\mathrm{m} / \mathrm{s})$ in axial cross sections

Fig. 8 - pressure, used full scale - the inlet pressure is penetrating into blind grooves, stopped at the groove bottom. In continuous holes, the pressure value is low, due to full conversion of the pressure into velocity.

Fig. 9 - velocity, used full scale - the main flow is going through holes, the flow through foam is very low (here not visible any details).
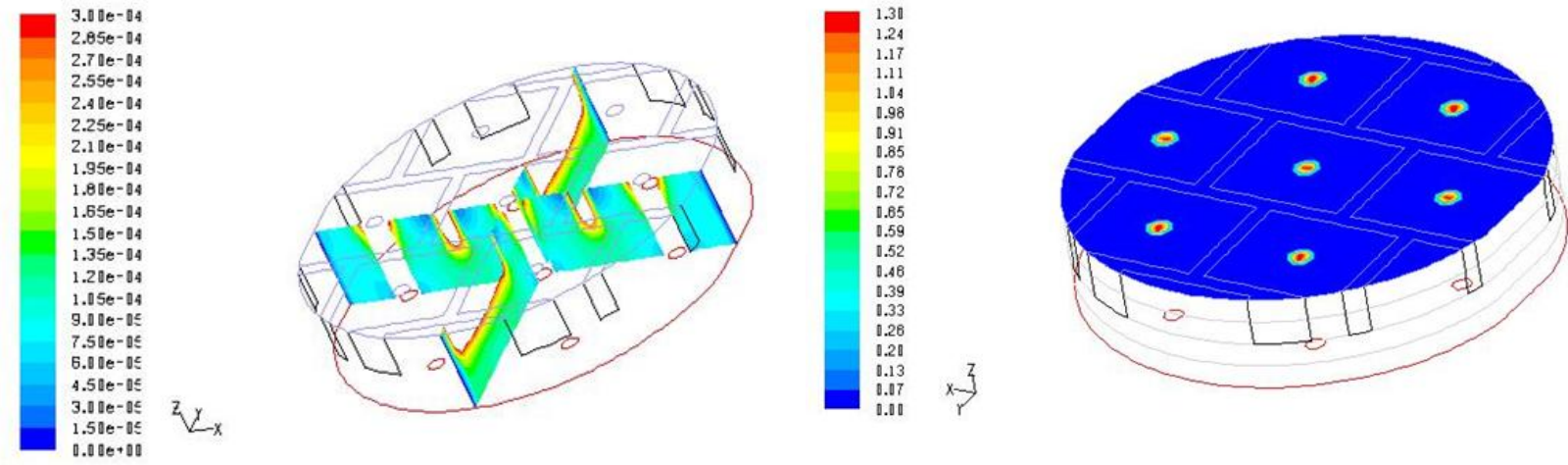

Fig. 10: Velocity field $(\mathrm{m} / \mathrm{s})$ in axial cross sections

Fig. 11: Velocity field $(\mathrm{m} / \mathrm{s})$ at the inlet plane

Fig. 10, velocity, used suppressed scale - the flow in grooves is stopped at the groove bottom and slightly is penetrating into foam volume. Simulation of full geometry can explain details of flow in permeable and perforated volume of foam.

Fig. 11 - velocity field $(\mathrm{m} / \mathrm{s})$ at the inlet plane, used full scale - maximum in holes, no details in the foam. 

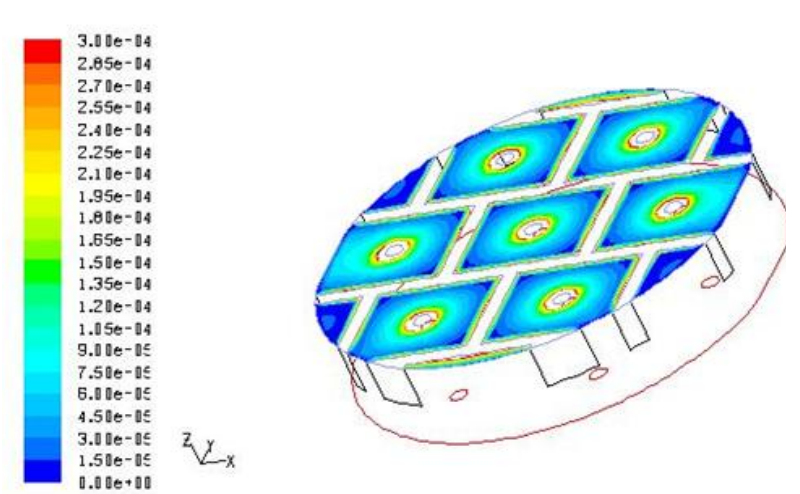
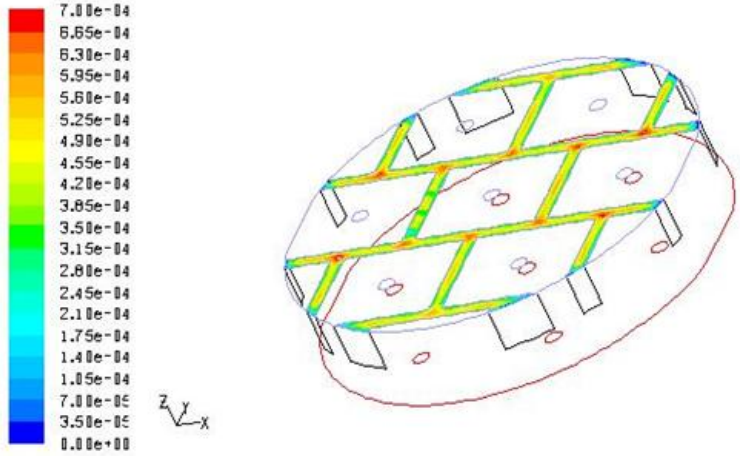

Fig. 12: Velocity field $(\mathrm{m} / \mathrm{s})$ at the inlet plane

Fig. 13: Velocity field $(\mathrm{m} / \mathrm{s})$ in grooves - inlet plane

Fig. 12 - velocity, used suppressed scale, at the inlet plane are visible details of the velocity field. Quick flows in holes induce slow flows in adjacent foam volume.

Fig. 13 - velocity, used suppressed scale, in grooves at the inlet plane the local maxima are observable in larger cross sections (in T-junctions); individual irregularities could be suppressed by finer mesh.
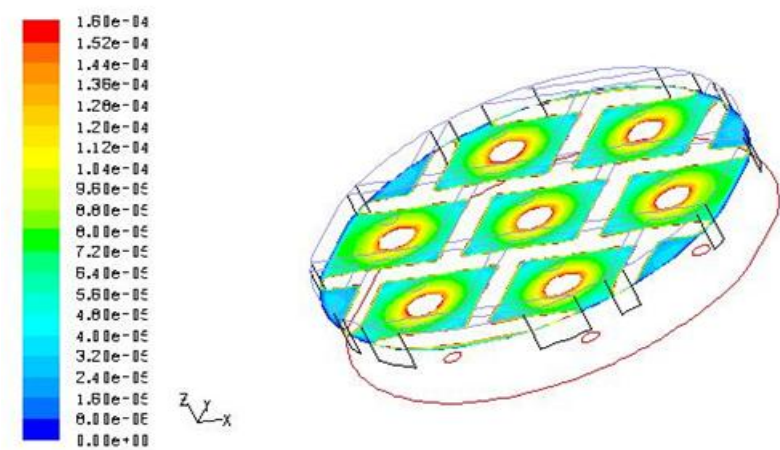

Fig. 14: Velocity field $(\mathrm{m} / \mathrm{s}) 7.5 \mathrm{~mm}$ from the inlet
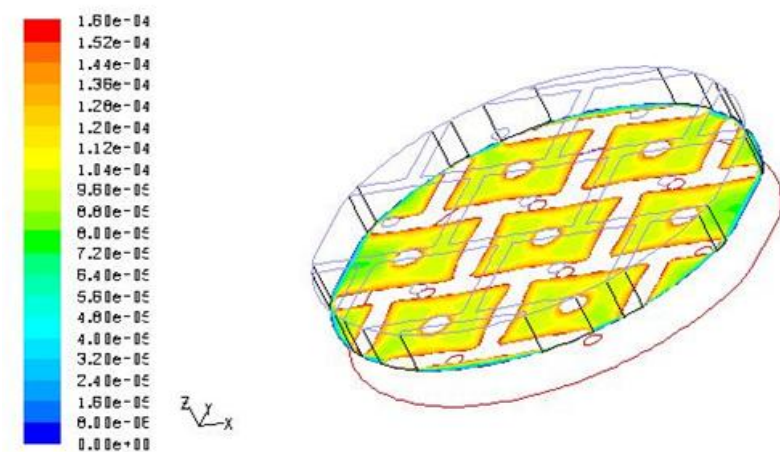

Fig. 15: Velocity field $(\mathrm{m} / \mathrm{s}) 15 \mathrm{~mm}$ from the inlet

Fig. 14 - velocity, used suppressed scale - in one half of the groove depth (at $7.5 \mathrm{~mm}$ ) is well visible, how quick flows in holes induce flows in adjacent foam volumes.

Fig. 15 - velocity, used suppressed scale at the groove bottom (depth of $15 \mathrm{~mm}$ ) - some local maxima at the sample periphery are effects of outer boundary condition. 

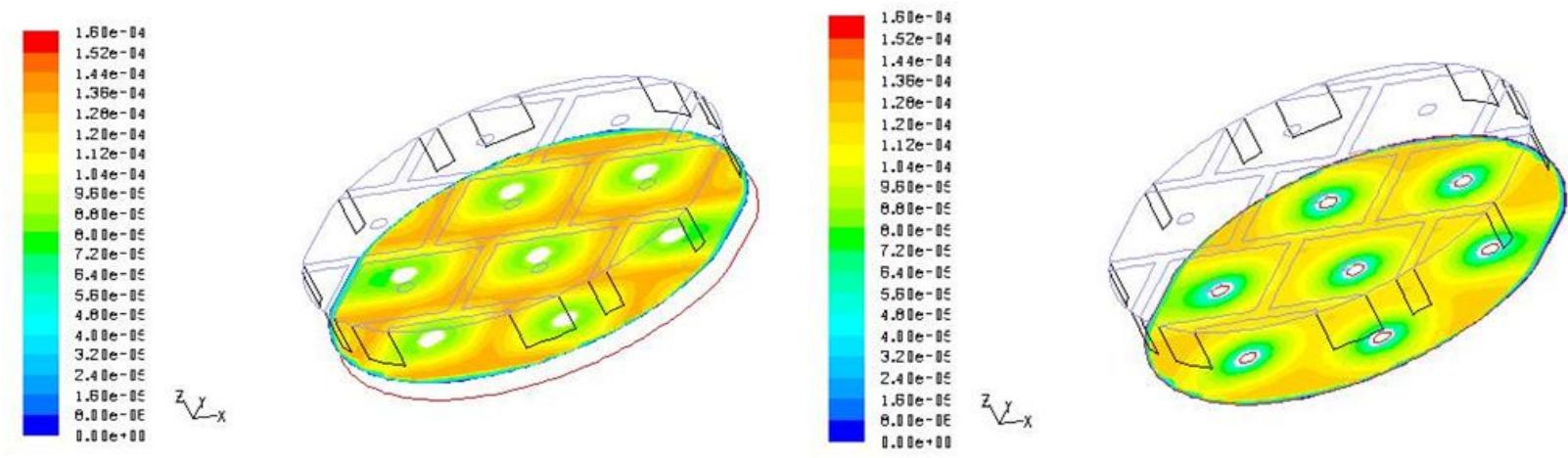

Fig. 16: Velocity field $(\mathrm{m} / \mathrm{s}) 22.5 \mathrm{~mm}$ from the inlet

Fig. 17: Velocity field $(\mathrm{m} / \mathrm{s})$ at the outlet

Fig. 16 - velocity, used suppressed scale - well visible induced flows in the foam around individual holes and relative stronger flows under individual grooves.

Fig. 17 - velocity, used suppressed scale - similar effects as above.

In general, in individual cross sections (Fig. 11 to Fig. 17) are visible some differences, but absolute values (order of $1 \mathrm{e}-5$ to $1 \mathrm{e}-4 \mathrm{~m} / \mathrm{s}$ ) can be neglected in comparison with flows in holes (up to $1.3 \mathrm{~m} / \mathrm{s}$ ). For presentation of fine flows suppressed scales should be used.
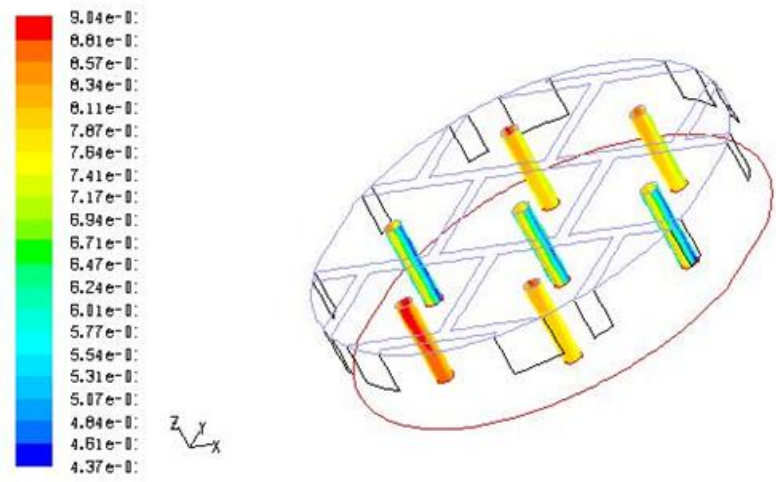

Fig. 18: Velocity field $(\mathrm{m} / \mathrm{s})$ along walls of individual holes

In the Fig. 18 wall velocities are not zero here (as on rigid walls), because those walls are permeable and some non-zero velocity is in foam volume, too. Real values of $0.3-0.7 \mathrm{~m} / \mathrm{s}$ are lower than axial maximum in the "empty" hole $(1.3 \mathrm{~m} / \mathrm{s})$. Higher velocity values are observed in peripheral holes, compared with middle holes. Maybe it is any influence of peripheral boundary condition, maybe in combination of relative coarse computation mesh.

\subsection{Reason of difference between measured and simulated flows}

Note 7: The checking mass balance in simulated cases above is OK, small differences between summary flows of inlet and outlet can be neglected. Even if the holes area is $1.1 \%$, only, of the whole sample area, the main flow of $99 \%$ approx. is going through holes and the flow through the foam $1 \%$ approx. It could be state that the foam is practically impermeable and used as elastic cushion, only.

However, measured flow is 6.82 times lower than simulated one. Generally said, the qualitative results (images of flow fields in the chapter 2.1), are all right, but the quantitative results of the mass balance (m/s, $\mathrm{kg} / \mathrm{s} \mathrm{etc}$.) are not correct. 
From speculative reasons the measured data were reduced in this rate, new permeability parameters were evaluated and modified simulation is realized once again. However, results were not modified enough.

Testing next various random values of permeability parameters, the total flow is not decreasing more. The flow through holes remains the same; the foam flow is increasing only a little. It is evident, that the main influence has the flow through holes (practically not influenced by different values of the foam permeability) and the influence of the flow through permeable foam is practically insignificant (can be increased a little by another value of the foam permeability). However, it is not possible to decrease the total flow 6.82 times, to get simulated flow values corresponding to the measured ones.

Therefore, the case containing both "empty" holes and "permeable" foam has not the resulting flow, corresponding to measured values.

To verify this hypothesis, simple model of "foam cake" (for both cases F0 and F1) was solved, too. Permeability parameters of such cake are used from the Tab. 3. For foam F1 it means, that measured permeability is any "averaged" permeability. Therefore in such model of the "foam cake" it is not possible to see details of the flow field in perforations, grooves, or foam, as in the section 2.1, but any "averaged" flow, only. Comparison of measured and simulated flows for foams F0 and F1 shows the Fig. 19.

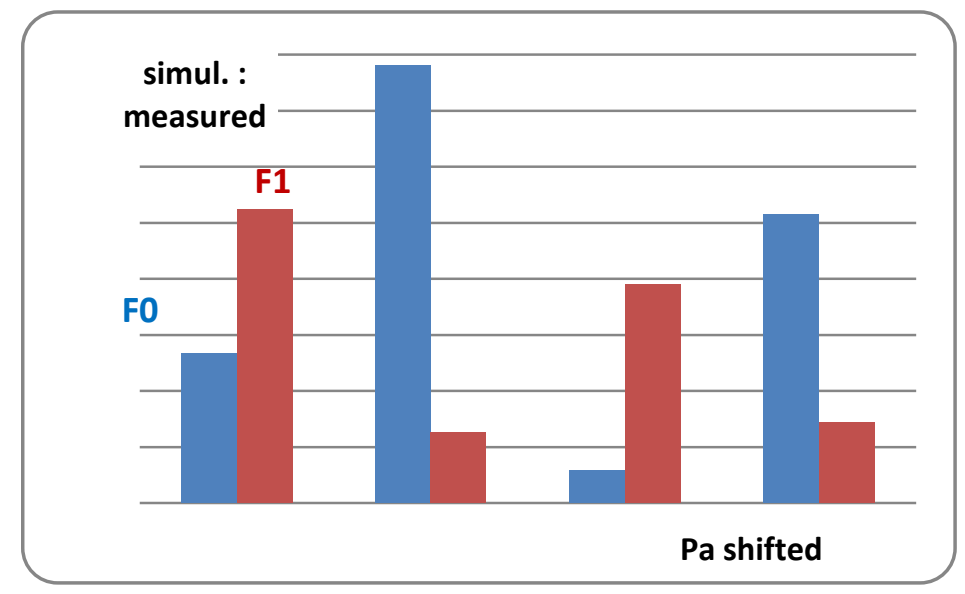

Fig. 19: Measured vs. simulated mass flows

Differences are in general in the range of $1 . .5 \%$, only, which is fully comparable with standard error of operational measuring devices of $4 \%$. The reason of detected error could be any numerical error, influence of relative coarse mesh etc. - not investigated more.

\subsection{Combination foam body + top fabric}

To the geometry in the section 2.1 above is simply added thin inlet area, to separate the inlet plane (pressure of 3 $\mathrm{Pa}$ ) and the plane of thin porous jump, which is defined just on the inlet plane of the foam body - in one plane cannot be defined two different boundary conditions.

As the top layer here are used three typical textiles from [1], in the Tab. 4 labelled as 1-1, 4-1 and 4-3, representing the smallest, medium and the highest air permeability with corresponding parameters of so-called porous jump (= pressure resistance in thin layer).

Table 4: Permeability of used top layers [1]

$\begin{array}{cccc}\text { top layer } & \mathrm{C} 2(1 / \mathrm{m}) & \alpha\left(\mathrm{m}^{2}\right) & \mathrm{t}(\mathrm{m}) \\ 1-1 & 4700000 & 1.54422 \mathrm{E}-11 & 0.0004 \\ 4-1 & 1659106 & 2.04836 \mathrm{E}-11 & 0.00041 \\ 4-3 & 222747 & 9.26330 \mathrm{E}-11 & 0.00054\end{array}$


The case without top layer is solved, too, to qualify the influence of slightly changed model by the added thin layer. Foam parameters are identical with the Tab. 3 above, the main results are following:

Without added layer: This case must be solved to test the influence of added thin inlet volume between boundary conditions of "inlet" and "porous jump". Results are similar to above mentioned basic case - the main part of air is flowing through holes (99\%), the foam flow is insignificant (1\%).

Adding the thin inlet layer the flow is of $24.4 \%$ higher. This difference is probably given by another geometry (and mesh) of compared cases, maybe by a little different permeability parameters, used in both models.

With added layer: Added layer decreases the total flow considerably, first in holes. The holes flow (both relative in $\%$ and absolute in $\mathrm{kg} / \mathrm{s}$ ) is now comparable with foam flow, from the foam + holes rate of $46 \%+54 \%$ up to the rate $30 \%+70 \%$, depending on the actual permeability of used top layer.

The foam flow is decreasing a little, only, from $91 \%$ to $96 \%$, depending on the actual permeability of used top layer. However, absolute value of this flow and its change can be neglected.

\subsection{Foam under load (pressed)}

This is the initial test of a real operation.

Hypothesis: Pressing the foam volume at $50 \%$ in the flow direction, the inner permeable cells are pressed at $50 \%$, too, so the permeability is decreasing at $50 \%$, too. In addition, it supposes that geometry deformation is along the axis of deformation, only, without any warping or buckling in cross directions.

Note 8:

Used simple hypothesis should be tested by measuring.

Special software is able to simulate high deformations of very elastic structures (foam, rubber,...). For it is necessary to evaluate material parameters (Young's module, Poisson's contraction).

For simple verification the model geometry remains the same (see the Fig. 7), new parameters of permeability are determined by the same manner as in the section 1.3 , from the condition of $50 \%$ of measured flow - see the Tab. 5 .

Table 5: Permeability parameters for pressed foam

$$
\mathrm{C} 2 \quad \alpha\left(\mathrm{m}^{2}\right) \quad 1 / \alpha\left(1 / \mathrm{m}^{2}\right)
$$

F0 $\quad 1.111 \mathrm{E}+10 \quad 7.052 \mathrm{E}-12 \quad 1.418 \mathrm{E}+11$

Comparison of the "pressed" results with original ones: Mass flows through foam and grooves are decreased on $28 \%$ and $27 \%$ respectively of original values. It means from $1 \%$ of the whole flow on $0.3 \%$ approx. It has not any influence on the flow through holes, because it represents $99 \%$ of the whole flow and some small flow changes in foam and grooves have not any influence.

\subsection{Permeability of perforated plate}

The influence of pressed foam thickness on air permeability should be tested experimentally. For measuring to use a firm perforated plate or sieve and to evaluate permeability of the plate alone and (plate + pressed foam) together. Then the flow through pressed foam is the difference between permeability for (foam+plate) and plate only - it is necessary to evaluate the influence of such added layer.

Permeability of perforated metal sheet of simple geometry, used for pressing of foam body during permeability measuring of pressed foam, could be both measured and simulated. 
As an example of the simulation, only, there is presented one possible case of the flow through perforated plate as follows:

perforation diameter of $6 \mathrm{~mm}$,

perforation pitches of $14 \mathrm{~mm}$,

sheet thickness of $2 \mathrm{~mm}$.

The plate is created as holes of $6 \mathrm{~mm}$ in hexahedral grid of $14 \mathrm{~mm}$, solved one element, only, for pressures of 0 1-2-3 Pa, resulting in mass flow (kg/s), which is recalculated into flow velocity (m/s). The Fig. 20 and Fig. 21 show the pressure field in hole between two volumes (inlet and outlet) and the corresponding velocity field.

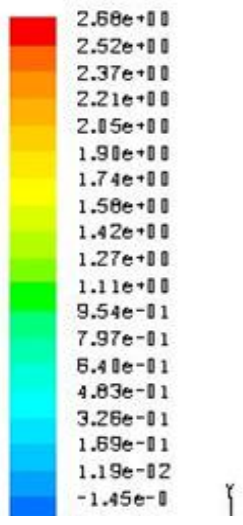

Fig. 20: Pressure field (Pa)- perforated sheet
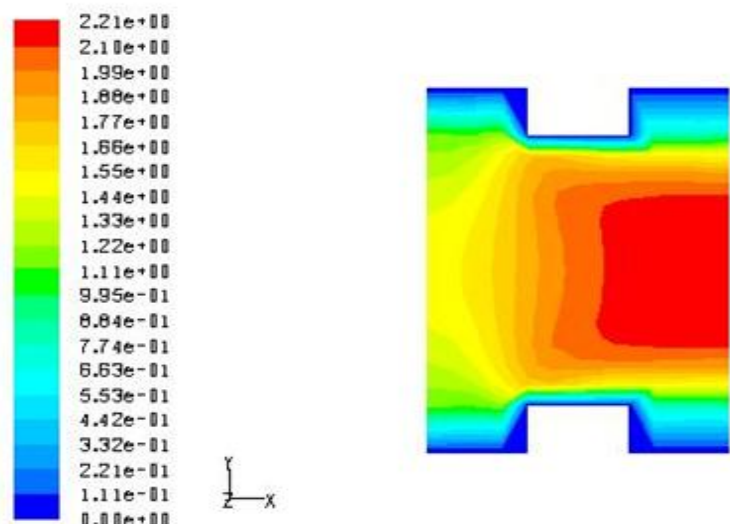

For next solution is used spare element of simple porous jump instead numerous holes in sheet. For testing is used the same model as in the section 2.3 (foam with added inlet layer).

The received mass flows are $84-87 \%$ of original mass flows - such coincidence is acceptable.

Next step is determination of permeability parameters for thin layer (porous jump); consisting from many holes, see the Tab. 6.

Table 6: Permeability parameters of perforated sheet

$$
\begin{array}{cr}
\mathrm{C} 2 & \alpha\left(\mathrm{m}^{2}\right) \\
1.631 \mathrm{E}+03 & 3.043 \mathrm{E}-07
\end{array}
$$

For the determination of perforated metal sheet influence on air permeability the parameters from the Tab. 6 are used for the model of porous jump, described in the section 2.3 above. Used layout of perforation decreases the flow through holes on $62 \%$, influence on foam flow in none. Measuring the permeability of pressed foam by any perforated sheet, it should be take into account both layers - foam body and pressing plate.

\section{CONCLUSION}

It is possible to state that method of numerical flow simulation, used for air permeability simulation through porous volumes, gives a good imagination about the quality and main features of the flow field in complicated volume combined from both continuous and blind air volumes and foam volumes (for instance the pressure or velocity distribution, concentration of the flow in the foam area around individual holes and grooves, flow spreading from the groove bottom in foam, flows in foam, induced by strong flow in adjoining holes etc.). 
However, absolute values of the mass flow in simulated cases of perforated and grooved foams are not correct they are several times different from measured data. Nevertheless, for qualitative comparison of various cases the method could be useful.

For given geometries the flow through foam volume represents typically $1 \%$ approx. only of the whole flow, the absolute majority is flowing through holes. Therefore, any changes of foam permeability cannot have any important influence on simulated value of total permeability in perforated and grooved case. It could be stated that strong flows through perforated holes have the main effect in total mass flow balance and the flow through foam is negligible.

A speculative change of permeability parameters do not have the expected effect. Testing the simple geometry of "foam cake" for permeability parameters of F0 and F1, the difference between mass flow measured and simulated is few percents, only. Such "averaged" permeability gives comparable results of simulation and measuring, but cannot display fine details of the flow field in the foam volume, first of all in the vicinity of perforations and grooves (if they are necessary, because the foam flow is $1 \%$ approx., only).

Zero flow at non-zero pressure (absolute term in the quadratic substitution $\mathrm{C} \neq 0$ ) is the result of any leakage, device insensitivity near zero, reading error etc. The reason of it could be determined, for the purpose of simulations the problem was here simply corrected by qualified "shifting" of measured values. Maybe, this could be the reason of different results of measuring and simulation, discussed above.

The flow resistance of added fabric layer (permeability parameters used after [1]) at the inlet side of the foam decreases the total flow considerably; the flow through holes is now similar with the flow through foam volume.

Influence of pressed foam layer has practically no influence on the total flow. The main part is flowing through "empty" holes practically without resistance (compared with reduced flow through the foam volume, due to the higher foam resistance after pressing). The pressing perforated sheet at the inlet side of the foam has an influence on the total flow through pressed sample.

From the practical point of view, the holes of very large diameters are not acceptable; their contours are marked through on the upholstery surface.

\section{ACKNOWLEDGMENT}

Our acknowledgment is given to VUTS Liberec - Center for Development in Machinery Research for the support in the framework of the grant NPU-LO1213 "National Program of Sustainability", granted by the Czech Ministry of Education, Youth and Sport.

\section{References}

[1] Adámek, K.: Car seat permeability - feasibility study, for KOD TU in Liberec, 2016, unpublished.

[2] Mazari, A.: Permeability measuring of thick foam layers - KOD FT TUL, 2016, 2017, unpublished

[3] Adámek, K.: Permeability of textile layers, Proc. of 15th International conference Applic. of exp. and numer. meth. in fluid mech.,TU in Žilina, Slovakia, 2008, 6 p.

[4] Software Fluent, actual version.

[5] Havrdová, M.: Evaluation of clothing textiles permeability (Příspěvek k hodnocení prodyšnosti oděvních tkanin). Dissertation FT TUL, 2004

[6]Buyuk Mazari, F., Adámek, K., Mazari A., Haveka A., Akcagun E.: Analysing theoretically the air flow through car seat foam material. Proc. of the IITAS 2017,7 p., Izmir, Turkey.

[7] Havelka, A., Glombiková, V., Mazari, F.B.: Monitoring thermophysiological comfort in the interlayer between driver and the carseat, Vlakna a Textil, 2015 (3-4), pp. 40-45.

The article contains our own data, only, using generally known physical laws and commercial both software and measuring devices. Therefore not more references were added. 